\title{
ON FIRMLY NON-EXPANSIVE MAPPINGS
}

\section{JOSEPH FRANK GORDON ${ }^{1, *}$, ESTHER OPOKU GYASI ${ }^{2}$}

\author{
${ }^{1}$ Department of Mathematics Education, Akenten Appiah-Menka University of Skills Training and \\ Entrepreneurial Development, Kumasi, Ghana \\ ${ }^{2}$ Department of Mathematical Sciences, University of Mines and Technology, Tarkwa, Ghana
}

${ }^{*}$ Corresponding author: josephfrankgordon@gmail.com

\begin{abstract}
In this paper, it is shown that for a closed convex subset $\mathcal{C}$ and to every non-expansive mapping $T: \mathcal{C} \rightarrow \mathcal{C}$, one can associate a firmly non-expansive mapping with the same fixed point set as $T$ in a given Banach space.
\end{abstract}

\section{INTRODUCTION}

The study of non-expansive mappings in the sixties have experimented a boost, basically motivated by Browder's work on the relationship between monotone operators, non-expansive mappings [1-3,3-5] and the seminal paper by Kirk [6], where the significance of the geometric properties of the norm for the existence of fixed points for non-expansive mappings was highlighted.

Now the history of firmly non-expansive mappings goes back to the paper by Minty [7], where he implicitly used this class of mappings to study the resolvent of a monotone operator. Browder [3] first introduced firmly non-expansive mappings in the concept of Hilbert spaces $\mathcal{H}$. That is, given a $\mathcal{C}$ closed convex subset of a Hilbert space $\mathcal{H}$, a mapping $F: \mathcal{C} \rightarrow \mathcal{H}$ is firmly non-expansive if for all $x, y \in \mathcal{C}$

$$
\|F x-F y\|^{2} \leq\langle x-y, F x-F y\rangle
$$

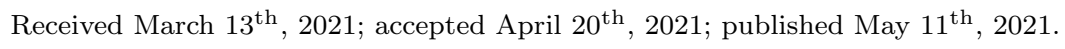

2010 Mathematics Subject Classification. 47H10, 54H25.

Key words and phrases. non-expansive mappings; firmly non-expansive mappings; metric projection; fixed points.

(C)2021 Authors retain the copyrights of their papers, and all open access articles are distributed under the terms of the Creative Commons Attribution License. 
In his study of non-expansive projections on subsets of Banach spaces, Bruck [8] defined a firmly nonexpansive mapping $F: \mathcal{C} \rightarrow \mathcal{X}$, where $\mathcal{C}$ is a closed convex subset of a real Banach space $\mathcal{X}$, to be a mapping such that for all $x, y \in \mathcal{C}$ and $\alpha \geq 0$,

$$
\|F x-F y\| \leq\|\alpha(x-y)+(1-\alpha)(F x-F y)\| .
$$

It is clear that equation (1.2) reduces to equation (1.1) when in Hilbert spaces and also when $\alpha=1, F$ becomes a non-expansive mappings, that is, for each $x$ and $y$ in $\mathcal{C}$, we have $\|F x-F y\| \leq\|x-y\|$. A trivial example of equation (1.2) is the identity mapping. A non-trivial example of equation (1.2) in a Hilbert space is given by the metric projection

$$
P_{\mathcal{C}} x=\operatorname{argmin}_{y \in \mathcal{C}}\{\|x-y\|\} .
$$

To see this, recall that in a real Hilbert space $\mathcal{H}, \forall x, y \in \mathcal{H}$, then $\langle x, y\rangle \geq 0$ if and only if

$$
\|x\| \leq\|x+a y\|
$$

for all $a \geq 0$. Now equation (1.2) can be written as

$$
\|F x-F y\| \leq\|F x-F y+\alpha(x-y-F x+F y)\| .
$$

Now applying equation (1.4) on equation (1.5), we obtain the following

$$
\begin{aligned}
\langle F x-F y, x-y-F x+F y\rangle & \geq 0, \\
\langle x-y-F x+F y, F x-F y\rangle & \geq 0, \\
\langle x-y-(F x-F y), F x-F y\rangle & \geq 0, \\
\langle x-y, F x-F y\rangle-\langle F x-F y, F x-F y\rangle & \geq 0, \\
\langle x-y, F x-F y\rangle & \geq\langle F x-F y, F x-F y\rangle, \\
\langle x-y, F x-F y\rangle & \geq\|F x-F y\|^{2} .
\end{aligned}
$$

Hence we have that in a real Hilbert space, a firmly non-expansive mapping $F$ can be written as

$$
\|F x-F y\|^{2} \leq\langle x-y, F x-F y\rangle
$$

But in a real Hilbert space, equation (1.3) satisfies the following inequality

$$
\left\|P_{\mathcal{C}} x-P_{\mathcal{C}} y\right\|^{2} \leq\left\langle x-y, P_{\mathcal{C}} x-P_{\mathcal{C}} y\right\rangle
$$

This means that from equations (1.6) and (1.7), we can simply conclude that $F=P_{\mathcal{C}}$ and so the metric projection $P_{\mathcal{C}}$ is a firmly non-expansive mapping in a real Hilbert space. 
In this paper, we give a simple proof showing that to any non-expansive self-mapping $T: \mathcal{C} \rightarrow \mathcal{C}$ that has fixed points, one can associate a large family of firmly non-expansive mappings having the same fixed point set as $T$. That is, from the point of view of the existence of fixed points on closed convex sets, non-expansive and firmly non-expansive mappings exhibit a similar behavior. However, this is no longer true in non-convex domains [9].

\section{Main Results}

Let $T$ be a non-expansive mapping defined on a closed convex subset $\mathcal{C}$ of a normed space $\mathcal{X}$, thus, $T: \mathcal{C} \rightarrow \mathcal{C}$. For a fixed $r \in \mathbb{R}_{>1}$, we can define the following mapping

$$
T_{r}: \mathcal{C} \rightarrow \mathcal{C} \quad \text { by } \quad x \mapsto\left(1-\frac{1}{r}\right) x+\frac{1}{r} T\left(T_{r} x\right) .
$$

Now we observe that equation (2.1) (the new mapping $T_{r}$ ) always exist. To see this, one can create an internal contraction $F: \mathcal{C} \rightarrow \mathcal{C}$ such that

$$
F(y)=\left(1-\frac{1}{r}\right) x+\frac{1}{r} T y, \quad \text { where } \quad x \quad \text { is fixed. }
$$

Now $\|F(y)-F(z)\|=\frac{1}{r}\|T y-T z\| \leq \frac{1}{r}\|y-z\|$. Hence $F$ is a contraction mapping and by the Banach contraction mapping theorem [10], there exists $u \in \mathcal{C}$ such that $F(u)=u$, thus, $u=\left(1-\frac{1}{r}\right) x+\frac{1}{r} T u$. Since for every $x \in \mathcal{C}$, we can find a unique $u$ such that $u=T_{r} x$, then equation (2.1) always exists. Now we have the following claims.

Claim 1: $T_{r}$ is a non-expansive mapping. To see this, we have the following:

$$
\begin{aligned}
\left\|T_{r} x-T_{r} y\right\| & =\left\|\left(1-\frac{1}{r}\right)(x-y)+\frac{1}{r}\left(T\left(T_{r} x\right)-T\left(T_{r} y\right)\right)\right\|, \\
& \leq\left(1-\frac{1}{r}\right)\|x-y\|+\frac{1}{r}\left\|T\left(T_{r} x-T\left(T_{r} y\right)\right)\right\|, \\
& \left.\leq\left(1-\frac{1}{r}\right)\|x-y\|+\frac{1}{r} \| T_{r} x-T_{r} y\right) \|, \\
\left\|T_{r} x-T_{r} y\right\|-\frac{1}{r}\left\|T_{r} x-T_{r} y\right\| & \leq\left(1-\frac{1}{r}\right)\|x-y\|, \\
\left(1-\frac{1}{r}\right)\left\|T_{r} x-T_{r} y\right\| & \leq\left\|\left(1-\frac{1}{r}\right)\right\|\|x-y\| .
\end{aligned}
$$

So we have that $T_{r}$ is a non-expansive mapping since $r>1$.

Claim 2: Now we prove that $T_{r} x$ is a firmly non-expansive mapping.

Now for $r>1, \alpha \in(0,1)$ and $\beta>0$, we have the following evaluation:

$$
\begin{aligned}
\left\|T_{r} x-T_{r} y\right\|= & \| \beta\left[\alpha(x-y)+(1-\alpha)\left(T_{r} x-T_{r} y\right)\right]-\beta \alpha(x-y) \\
& +\left(T_{r} x-T_{r} y\right)-\beta(1-\alpha)\left(T_{r} x-T_{r} y\right) \| .
\end{aligned}
$$


But

$$
\begin{aligned}
\left(T_{r} x-T_{r} y\right)-\beta(1-\alpha)\left(T_{r} x-T_{r} y\right)= & \left(1-\frac{1}{r}\right)(x-y)+\frac{1}{r}\left(T\left(T_{r} x\right)-T\left(T_{r} y\right)\right) \\
& -\beta(1-\alpha)\left[\left(1-\frac{1}{r}\right)(x-y)+\frac{1}{r}\left(T\left(T_{r} x\right)-T\left(T_{r} y\right)\right)\right], \\
= & \left(1-\frac{1}{r}\right)(x-y)-\beta(1-\alpha)\left(1-\frac{1}{r}\right)(x-y)+\frac{1}{r}\left(T\left(T_{r} x\right)-T\left(T_{r} y\right)\right) \\
& -\beta(1-\alpha) \frac{1}{r}\left(T\left(T_{r} x\right)-T\left(T_{r} y\right)\right), \\
= & \left(1-\frac{1}{r}\right)(x-y)[1-\beta(1-\alpha)] \\
& +\frac{1}{r}(1-\beta(1-\alpha))\left(T\left(T_{r} x\right)-T\left(T_{r} y\right)\right) .
\end{aligned}
$$

Hence

$$
\begin{aligned}
\left\|T_{r} x-T_{r} y\right\|= & \| \beta\left[\alpha(x-y)+(1-\alpha)\left(T_{r} x-T_{r} y\right)\right]-\beta \alpha(x-y)+\left(1-\frac{1}{r}\right)(x-y)[1-\beta(1-\alpha)] \\
& +\frac{1}{r}(1-\beta(1-\alpha))\left(T\left(T_{r} x\right)-T\left(T_{r} y\right)\right) \|, \\
= & \| \beta\left[\alpha(x-y)+(1-\alpha)\left(T_{r} x-T_{r} y\right)\right]+\left[-\beta \alpha+\left(1-\frac{1}{r}\right)(1-\beta(1-\alpha))\right](x-y) \\
& +\frac{1}{r}\left(1-\beta(1-\alpha)\left(T\left(T_{r} x\right)-T\left(T_{r} y\right)\right) \| .\right.
\end{aligned}
$$

Now let $-\beta \alpha+\left(1-\frac{1}{r}\right)(1-\beta(1-\alpha))=0$. This implies that

$$
\begin{aligned}
\beta & =\frac{r-1}{\alpha r+(\alpha-1)(r-1)}, \\
\frac{1}{r}(1-\beta(1-\alpha)) & =\frac{\alpha}{\alpha r+(1-\alpha)(r-1)} .
\end{aligned}
$$

Hence we have

$$
\left\|T_{r} x-T_{r} y\right\| \leq \beta\left\|\alpha(x-y)+(1-\alpha)\left(T_{r} x-T_{r} y\right)\right\|+\frac{\alpha}{\alpha r+(1-\alpha)(r-1)}\left\|T\left(T_{r} x\right)-T\left(T_{r} y\right)\right\| .
$$

So by the non-expansiveness of $T$, The above inequality becomes

$$
\begin{aligned}
\left\|T_{r} x-T_{r} y\right\| \leq & \beta\left\|\alpha(x-y)+(1-\alpha)\left(T_{r} x-T_{r} y\right)\right\|+\frac{\alpha}{\alpha r+(1-\alpha)(r-1)}\left\|T_{r} x-T_{r} y\right\|, \\
= & \frac{r-1}{\alpha r+(1-\alpha)(r-1)}\left\|\alpha(x-y)+(1-\alpha)\left(T_{r} x-T_{r} y\right)\right\| \\
& +\frac{\alpha}{\alpha r+(1-\alpha)(r-1)}\left\|T_{r} x-T_{r} y\right\| .
\end{aligned}
$$


After simplifying we obtain the following results

$$
\begin{aligned}
\left\|T_{r} x-T_{r} y\right\|-\frac{\alpha}{\alpha r+(1-\alpha)(r-1)}\left\|T_{r} x-T_{r} y\right\| & \leq \frac{r-1}{\alpha r+(1-\alpha)(r-1)}\left\|\alpha(x-y)+(1-\alpha)\left(T_{r} x-T_{r} y\right)\right\|, \\
{\left[1-\frac{\alpha}{\alpha r+(1-\alpha)(r-1)}\right]\left\|T_{r} x-T_{r} y\right\| } & \leq \frac{r-1}{\alpha r+(1-\alpha)(r-1)}\left\|\alpha(x-y)+(1-\alpha)\left(T_{r} x-T_{r} y\right)\right\|, \\
\frac{r-1}{\alpha r+(1-\alpha)(r-1)}\left\|T_{r} x-T_{r} y\right\| & \leq \frac{r-1}{\alpha r+(1-\alpha)(r-1)}\left\|\alpha(x-y)+(1-\alpha)\left(T_{r} x-T_{r} y\right)\right\|, \\
\left\|T_{r} x-T_{r} y\right\| & \leq\left\|\alpha(x-y)+(1-\alpha)\left(T_{r} x-T_{r} y\right)\right\|,
\end{aligned}
$$

since $\beta=\frac{r-1}{\alpha r+(1-\alpha)(r-1)}>0$. Hence $T_{r}$ is firmly non-expansive mapping.

Claim 3: We have that $z$ is a fixed point of $T$ if and only if it is also a fixed point of $T_{r}$.

Proof. Now suppose that $T z=z$. Then we have the following evaluation:

$$
\begin{aligned}
\left\|T_{r} z-z\right\| & =\left\|\left(1-\frac{1}{r}\right) z+\frac{1}{r} T\left(T_{r} z\right)-z\right\|, \\
& =\left\|\frac{1}{r} T\left(T_{r} z\right)-\frac{1}{r} z\right\|, \\
& =\frac{1}{r}\left\|T\left(T_{r} z\right)-z\right\|, \\
& =\frac{1}{r}\left\|T\left(T_{r} z\right)-T z\right\|, \\
& \leq \frac{1}{r}\left\|T_{r} z-z\right\| .
\end{aligned}
$$

Hence $\left\|T_{r} z-z\right\| \leq \frac{1}{r}\left\|T_{r} z-z\right\|$ which is not possible since $r>1$. it is possible when $\left\|T_{r} z-z\right\|=0 \Rightarrow T_{r} z=z$. So $z$ is a fixed point of $T_{r} z$. On the other hand, let us suppose that $T_{r} z=z$, that is $z$ is a fixed point of $T_{r}$. Then

$$
\begin{aligned}
z & =T_{r} z, \\
& =\left(1-\frac{1}{r}\right) z+\frac{1}{r} T\left(T_{r} z\right), \\
& =\left(1-\frac{1}{r}\right) z+\frac{1}{r} T(z) .
\end{aligned}
$$

This gives us

$$
\left[1-\left(1-\frac{1}{r}\right)\right] z=\frac{1}{r} T(z)
$$

Hence $z$ is a fixed point of $T$ and that concludes our main result.

Conflicts of Interest: The author(s) declare that there are no conflicts of interest regarding the publication of this paper. 


\section{REFERENCES}

[1] F.E. Browder, Nonlinear monotone operators and convex sets in banach spaces. Bull. Amer. Math. Soc. 71(5) (1965), 780-785.

[2] F.E. Browder, Nonexpansive nonlinear operators in a Banach space. Proc. Nat. Acad. Sci. USA, 54(4) (1965), $1041-1044$.

[3] F.E. Browder, Convergence theorems for sequences of nonlinear operators in banach spaces. Math. Zeitschr. 100(3) (1976), 201-225.

[4] F.E. Browder, W.V. Petryshyn. The solution by iteration of nonlinear functional equations in banach spaces. Bull. Amer. Math. Soc. 72(3) (1966), 571-575.

[5] F.E. Browder, W.V. Petryshyn. Construction of fixed points of nonlinear mappings in Hilbert space. J. Math. Anal. Appl. 20(2) (1967), 197-228.

[6] W. Kirk. A fixed point theorem for mappings which do not increase distances. Amer. Math. Mon. 72(9) (1965), $1004-1006$.

[7] J. George, Minty et al. Monotone (nonlinear) operators in Hilbert space. Duke Math. J. 29(3) (1962), 341-346.

[8] R. Bruck. Nonexpansive projections on subsets of Banach spaces. Pac. J. Math. 47(2) (1973), 341-355.

[9] R. Smarzewski. On firmly nonexpansive mappings. Proc. Amer. Math. Soc. 113(3) (1991), 723-725.

[10] S. Banach, H. Steinhaus. Sur le principe de la condensation de singularités. Fundam. Math. 1(9) (1927), 50-61. 